\title{
Now: A Discourse-Based Theory
}

\author{
Julie Hunter \\ l'Université de Pau et des Pays de l'Adour and \\ l'EHESS/l'Institut Jean-Nicod, Paris \\ juliehunter@gmail.com
}

\begin{abstract}
This paper offers a semantic theory of uses of now in which now refers to a time introduced in discourse. I argue that the interpretation of an anaphoric use of now is determined by the rhetorical structure of the discourse in which the token of now figures.
\end{abstract}

Keywords. anaphora, now, rhetorical structure, discourse structure

In general, now is interpreted as the utterance time and cannot refer to a time made salient in the discourse in the way that a third person pronoun can refer to an individual made salient in the discourse:

(1) I like to think about my grandmother. I always had a great time with her.

(2) I like to think back on the summer of '97. I was so happy *now.

Yet there are exceptions (cf. Banfield 1982, Hunter 2010, Kamp \& Reyle 1993, Lee \& Choi 2009, Predelli 1998, Recanati 2004, Schlenker 2004). In the following examples, now denotes a time that lies in the past of the utterance time and is introduced at some prior point in the discourse:

(3) Five months later, I sat with her as she lay in bed, breathing thin slivers of breath and moaning... I was alone in her bleak room. Alone, because there was none of her in it, just a body that now held no essence of my mum. ${ }^{1}$

(4) The letter is marked "personal and private" and is addressed to President Franklin D. Roosevelt's secretary, Grace Tully, who was with the ailing chief executive in Warm Springs, Ga., that Thursday in 1945. The writer was Lucy Mercer Rutherfurd, who decades before had been FDR's mistress and who now was making arrangements for what would be their last fateful meeting at the president's rural retreat. ${ }^{2}$

(3) is taken from an article in which the author describes her mother's struggles with Alzheimer's. Throughout the article, it is clear that the author is recounting past events. Her use of now does not denote the utterance time in any sense; it rather denotes a time in the past at which she visited her ailing mother. The two sentences in (4) are about a letter to FDR that was acquired by the National

\footnotetext{
1 'Her misery was now so deep, her existence so shallow - Fiona Phillips on dealing with Alzheimer's', from Daily Mail, 28.08.2010.

2 'What was for FDR's eyes only is now for yours', The Washington Post, 29.07.2010.
} 
Archives. The author of the article describes the writing of the letter as an event in the past and clearly distances that event from the time of the acquisition. Still, he can use now to denote the time of the past letter writing event.

This paper offers a semantic theory of anaphoric uses of now; that is, uses of now in which now refers to a time introduced in discourse. Contrary to existing theories of now, I argue that the interpretation of an anaphoric use of now is determined by the rhetorical structure of the discourse in which the token of now figures. The details of my theory are presented in Segmented Discourse Representation Theory (Asher \& Lascarides 2003).

\section{Previous discourse accounts}

Kamp \& Reyle (1993) recognize that now can be used anaphorically to refer to a time introduced in discourse. However, now can only be so used, they claim, to modify past tense clauses that describe states. The idea underlying this distinction is that clauses describing states exploit the incoming reference time while clauses describing events introduce reference times of their own. While I cannot elaborate on Kamp \& Reyle's view here, the important point is that because state-denoting clauses exploit incoming reference points, they are able to shift the temporal perspective point (TPpt) of a discourse, where the TPpt is the time relative to which events are described as unfolding. Event denoting sentences, because they do not exploit incoming reference times, do not shift the TPpt. As now depends on the TPpt of a discourse, it can refer to a past time only when the TPpt has been shifted to the past by a state-denoting sentence.

Kamp \& Reyle's account does not do justice to the data on now, however. First, now can be used to modify event denoting clauses.

(5) Before being dipped into the liquid air, it had not caught fire; but now it exploded, it was consumed so rapidly. ${ }^{3}$

A second problem stems from a claim that clauses that describe events shift the time relative to the input context while clauses that describe states inherit the time of the incoming context. A third problem is that Kamp \& Reyle use a discourse theory that updates the TPpt for a discourse sentence by sentence, or perhaps clause by clause, without taking into account the relations between these sentences or clauses. ${ }^{4}$ What we see when we look at data on now is that (a) a past tense clause modified by now may fail to stand in a temporal relation to the clause that has come before it, regardless of its aspect, and (b) even if there is a relation between the two clauses, this relation may not be enough to determine the interpretation of now because sometimes the time denoted by now

\footnotetext{
${ }^{3}$ Variation on example in 'Liquid Air Experiments,' The New York Times, 13.05.1899.

${ }^{4}$ Lee \& Choi (2009) also suffers from the second and third problems for Kamp \& Reyle's view. Their treatment of aspect is more nuanced than Kamp \& Reyle's, but their account retains the division between state and event denoting clauses as well as a discourse theory that simply updates the reference point clause by clause.
} 
is the time introduced by a clause much earlier in the discourse. Such long distance relationships can hold regardless of the aspect of the now-modified clause.

When $t_{\beta}$ is independent of $t_{\alpha}$ : Kamp \& Reyle hold that the time denoted by a clause $\beta$ depends on the tense and aspect of $\beta$ together with a reference point introduced by the previous sentence. If $\alpha$ is the clause preceding $\beta, \beta$ should stand in a temporal relation with $\alpha$. Yet sometimes, a clause immediately preceding $\beta$ can denote a time that is completely irrelevant to the interpretation of $\beta$.

(6) Asked in 2012

a. [Why was the left so much more accepting of the 2011 budget than of the 2010 budget? $]_{\chi}$

b. [First, many on the left took a cue from conservatives, $]_{\gamma}$ [who had assailed the 2011 budget as falling short of the cutting that was needed. $]_{\alpha}$

c. [Second, Mr. Obama was now in better standing with liberals than he had been in 2010] $]_{\beta}$ [having recently repealed "don't ask, don't tell". $]_{\eta}$

Kamp \& Reyle predict that because now modifies a stative clause in $\beta$, it should refer to the temporal perspective point determined by the discourse through clause $\alpha$. Because $\alpha$ is in the past perfect, it in turn exploits the time introduced by the simple past clause $\gamma$, thereby making $t_{\gamma}$ (the time denoted by $\gamma$ ) the temporal perspective point. $t_{\gamma}$, of course, must have started before $t_{\chi}$ - the left first took the cue and then accepted the budget - but given that it comes after the conservative reaction to the president's budget, we know that $t_{\gamma}$ did not begin too long before the beginning of $t_{\chi}$. (I assume the question presupposes that the left was more accepting of the 2011 budget.) Intuitively, $t_{\beta}$ is independent of $t_{\alpha}$ and, therefore, $t_{\gamma}$; now refers to the time at which the left accepted the budget not the time at which they took a cue from conservatives. The tense and aspect of the sentences in (6) do not alone determine a temporal relation between $\gamma$ (or $\alpha$ ) and $\beta$, though the discourse does enforce a temporal relation between $\beta$ and $\chi: t_{\beta}$ overlaps $t_{\chi}$ and because $\beta$ is presented as an answer, or in this case an explanation, of $\chi, t_{\beta}$ must begin before the beginning of $t_{\chi}$. If we view (6) as a question with multiple independent answers, as opposed to a mere sequence of sentences, the temporal relation between $\beta$ and $\chi$ on the one hand coupled with the temporal independence of $\gamma / \alpha$ and $\beta$ on the other is no surprise.

(7) demonstrates the same point using a different rhetorical structure.

(7) a. [When Mr. Kaine agreed to run the DNC in 2009 $]_{\chi}$ - [even while finishing his last year as governor $]_{\gamma}$ - [his closest advisers were stunned $]_{\eta}$ [and they counseled him to renege. $]_{\alpha}$

b. [Now Mr. Kaine was facing an unwanted repeat of the same, uncomfortable situation.] ${ }^{5}$

Again, because $\beta$ describes a state using the past perfect, it should set the time of $\alpha$ as the temporal perspective point and then inherit that time. Yet intuitively,

\footnotetext{
${ }^{5}$ Variation on example from 'Will Obama Ask Kaine to Seek Virginia Senate Seat?', The New York Times, 10.02.2011.
} 
now in $\beta$ refers to a time introduced in the discourse prior to the introduction of clauses $\chi-\alpha$, which describe eventualities holding well in the past of $t_{\beta}$.

When $t_{\beta}$ is the time denoted by a clause preceding $\alpha$ : Even when a clause $\beta$ is temporally related to the preceding clause $\alpha$, Kamp \& Reyle can still fail to make the right predictions. They predict, for example, that event denoting clauses in the simple past, like $\beta$ in (8) below, will denote a time other than that denoted by the preceding clause. While this is the case in (8), Kamp \& Reyle miss the stronger claim that now refers to $t_{\chi}$.

(8) a. [The scientist dipped the felt into liquid air $]_{\gamma}$ [and the result was astonishing $]_{\chi}$.

b. [Before being dipped into the liquid air, it had not caught fire; $]_{\alpha}$ [but now it exploded, it was consumed so rapidly. $]_{\beta}$

While Kamp \& Reyle are right that a past tense use of now must refer to a time already available in the discourse and that this time will not in general be made available by the preceding clause if the now modified clause denotes an event, (8) shows that now need not find its referent in the preceding clause. Kamp \& Reyle's prediction that now cannot modify past tense clauses describing past events - a prediction discredited by examples like (8) - is explained in part by the fact that they only considered the temporal relation between a clause $\beta$ and the temporal perspective point used by the previous clause $\alpha$.

\section{Rhetorical contexts and now}

The temporal relations in a text-which determine the time to which a past use of now will refer-are not determined, at least not entirely, by the tense and aspect of individual sentences together with the order in which they appear in the text. To make predictions about the interpretation of now in past tense clauses, we need a theory that allows a clause $\beta$ to stand in a temporal relation to a clause $\chi$ even if there is a clause $\alpha$ introduced in the discourse between $\chi$ and $\beta$ such that (a) $\alpha$ stands in no temporal (or rhetorical) relation to $\beta$ or (b) the temporal or rhetorical relation between $\alpha$ and $\beta$ is not sufficient for determining the time of $\beta$. We need the structure offered by a theory of rhetorical contexts and relations between clauses in a discourse. I will show that in particular, the temporal relations offered by Segmented Discourse Representation Theory (Asher \& Lascarides 2003), or SDRT, can be used to make more accurate predictions about the interpretation of now than can theories of tense and aspect alone.

To capture the semantics of now, we need a theory of discourse content that uses structured contexts. I begin with Kamp \& Reyle's Discourse Representation Theory and add structure to discourse contexts in two ways. First, to each DRS, I add a level, call it $\mathrm{K}_{0}$, that represents information about utterance events. $\mathrm{K}_{0}$ is the most global level of a given DRS K; the content of utterances, i.e., the content that is normally treated by discourse theories like DRT, is added to sub-contexts of this 'extra-linguistic' level. The notion of $\mathrm{K}_{0}$ is introduced in Hunter (2010) in order to handle indexicals, among other expressions, and we 
need it to handle examples in which now picks out the time of utterance rather than a time introduced in discourse. ${ }^{6}$

Second, I expand on Hunter (2010)'s contexts by adding rhetorical structure as developed by Asher \& Lascarides (2003) and use Asher \& Lascarides' semantics for discourse relations. We start by dividing a discourse into elementary discourse units or EDUs, where an EDU is a minimal unit in a discourse that can stand in a rhetorical relation with another unit - EDUs are, in a sense, the 'words' of a discourse. Next, each EDU is represented as a DRS. Finally, each DRS for each EDU is related to another EDU, or chunk of EDUs, via a rhetorical relation. Both the content of the segmented DRSs and the relations between them are recorded in our contexts below level $\mathrm{K}_{0}$.

SDRT represents the rhetorical structure of a discourse in a two-dimensional graph space that distinguishes between subordinating and coordinating relations. In a subordinating relation, $\operatorname{suB}(\alpha, \beta), \beta$ does not move the discourse forward but simply provides more information about $\alpha$, in the form of, e.g., background, explanation or elaboration. In a coordinating relation, $\operatorname{COOR}(\alpha, \beta), \beta$ does not provide more information about $\alpha$ but rather moves the discourse forward relative to a topic shared by $\alpha$ and $\beta$.

With our structured discourse contexts in place, I propose that we treat now as an anaphoric, presuppositional expression along the lines of van der Sandt (1992). While I will not provide a complete motivation for this proposal here - see Hunter (2010), Hunter \& Asher (2005), Maier (2009), Roberts (2002), and Zeevat (1999) for arguments - the general idea is that now triggers a presupposition that must be bound to, or otherwise satisfied by, an antecedent time. Now, like other indexicals, does not bring along its own interpretation, but depends on the incoming context - generally the $\mathrm{K}_{0}$ level of the incoming context - to provide one. The fact that it is up to the incoming context to provide an antecedent for standard indexicals is seen most clearly with you and that, which can fail to refer if the context does not provide an antecedent.

Now can find its antecedent time either from the extra-linguistic context, $\mathrm{K}_{0}$, or from the discourse context, $\mathrm{K}_{1}-\mathrm{K}_{n}$. Now exhibits a strong preference for resolution to the utterance time, but this preference can be over-ridden when resolution at $\mathrm{K}_{0}$ is blocked. In the examples considered here, the past tense blocks resolution at the extra-linguistic level. To capture now's preference for resolution at $\mathrm{K}_{0}$, I use the operator $\uparrow$ introduced in Hunter \& Asher (2005), which requires material in its scope to be resolved at the highest context possible. Given DRT's treatment of existential formulas as introducers of discourse referents, the presupposition of now will look like this:

(9) $\uparrow \exists t(t=$ ?)

'?' signals that $t$ is anaphoric and must be identified with a discourse referent for a time already available in the context in which now's presupposition is triggered.

Now, like other indexicals and third person pronouns, is incapable of local accommodation. It requires that there be a super-ordinate time that it can treat

\footnotetext{
${ }^{6}$ See Hunter (2010) and Maier (2009) for a motivation of such structured contexts.
} 
as the 'current' time. Even when it refers to the utterance time, its presupposition is triggered in a sub-context of $\mathrm{K}_{0}$ and then bound in $\mathrm{K}_{0}$. When now cannot be resolved to the utterance time, I claim that it is resolved to the time of its immediately super-ordinate antecedent clause. That is, when $\beta$ in a subordinating relation $\operatorname{SUB}(\alpha, \beta)$ is modified by now, the time denoted by now will be determined by the time of the eventuality described in $\alpha$ such that the relation between the two times will be as close to identity as possible given the semantics of the rhetorical relation relating $\alpha$ and $\beta$. As all subordinating relations in SDRT would allow for temporal overlap between $\alpha$ and $\beta$, temporal overlap will be required when $\beta$ is modified by now. For ELABORATION and BACKGROUND, which require that one of their arguments be temporally included in the other, the addition of now will require identity between the times denoted by the arguments. For EXPLANATION, identity is ruled out: a cause must begin before its effect. The addition of now will, however, entail that the cause started right before its effect. These features of now's semantics have the following consequences:

Now restricts the temporal relations predicted by SDRT: Now restricts the temporal relations that a theory of rhetorical structure like SDRT predicts will hold between a subordinate clause and its super-ordinate antecedent. For example, if a clause $\beta$ explains a clause $\alpha$, SDRT allows that the time of $\beta\left(t_{\beta}\right)$ might start well before $t_{\alpha}$ and it might even end before $t_{\alpha}$ begins.

(10) $[\text { I hit him today }]_{\alpha}$ [because he hit me last week. $]_{\beta}$

In (10), the event described in $\beta$ ended before that described in $\alpha$ began. If $\beta$ is modified by now, however, $t_{\beta}$ must overlap $t_{\alpha}$ and must start just before $t_{\alpha}$.

(11) $[\text { I hit him }]_{\alpha}$ [because he $\left({ }^{*}\right.$ now $)$ hit me. $]_{\beta}$

(12) a. [This became apparent in Darwin's reaction to Jenkin's critique $]_{\gamma} \ldots$

b. [Darwin gave up his original assumption that evolution occurred best in small, isolated populations $]_{\chi}$,

c. [because he now feared that small populations would not throw up enough individual variants for selection to be effective. $]_{\beta}$

In (11), the cause ended before the effect began, so now is not licensed. In (12), the cause $(\beta)$ immediately brought about its effect $(\chi)$ and $t_{\chi}$ overlaps $t_{\beta} \cdot \chi$ and $\beta$ together elaborate on Darwin's reaction to Jenkin's critique, introduced in $\gamma$. The semantics of ELABORATION require that $t_{\chi}$ and $t_{\beta}$ together be included in $t_{\gamma}$.

$\operatorname{COOR}(\alpha, \boldsymbol{n o w}-\beta)$, then $\operatorname{SuB}(\chi,(\alpha, \boldsymbol{n o w}-\beta))$ : If a past-tense, now-modified clause $\beta$ is related to a preceding clause $\alpha$ via a coordinating relation, the whole unit $(\alpha+\beta)$ will be subordinate to another clause $\chi$ whose time will serve as now's antecedent. ${ }^{7}$ In (4), $\alpha$ and $\beta$ provide information about the same individual and so would be related via CONTINUATION, a coordinating relation in SDRT, while the unit $\alpha+\beta$ would be related to $\chi$ by BACKGROUND, a subordinating relation.

\footnotetext{
7 Now can be used as a modifier of past tense sentences in narratives without an
} explicit super-ordinate antecedent: 
(4) a. [The letter is marked "personal and private" and is addressed to... $]_{\eta}$

b. [The writer was Lucy Mercer Rutherfurd, $]_{\chi}$

c. [who decades before had been FDR's mistress] $]_{\alpha}$ [and who now was making arrangements for what would be their last fateful meeting at the president's rural retreat. $]_{\beta}$

The semantics of BACKGROUND in SDRT allow that if a clause $\beta$ is subordinate to a clause $\chi$ via BACKGROUND, then $t_{\beta}$ can start well before $t_{\chi}$. Unlike EXPLANATION, however, $t_{\chi}$ must always be included in $t_{\beta}$. When we add now to $\beta$, temporal overlap is taken care of by the semantics of BACKGROUND, but $t_{\beta}$ must start when $t_{\chi}$ starts. Because $\chi$ elaborates on or provides background for $\eta$ by providing information about who wrote the letter, $\chi$ in turn inherits $\eta$ 's time. I assume that since $\chi$ and $\eta$ are both about the writing of the letter under discussion, the time that they both denote is the time of the letter writing event. Now is thus interpreted as the time of the letter writing event, as desired.

Now can be used to enforce a temporal break: Suppose a now-modified clause $\beta$ is in a coordinating relation with a clause $\alpha$ where $\alpha+\beta$ is subordinate to another clause $\chi$ as described above. If the time of $\alpha$ is different from the time of $\chi$ and the rhetorical relation between $\alpha$ and $\beta$ does not enforce a temporal break between $\alpha$ and $\beta$ then now will be licensed to enforce a break and a return to the time of $\chi$. The felicity of (4), for example, is greatly aided by now because CONTINUATION does not impose a temporal relation between its arguments. With now, it is clear that while on the one hand, the now-modified clause is still providing background, it's providing information about what was going on at the time of the letter writing event (indirectly) introduced in $\chi$, not at the time of $\alpha$. Similar remarks can be made for (8):

(8) a. [The scientist dipped the felt into liquid air $]_{\gamma}[\text { and the result was astonishing }]_{\chi}$.

b. [Before being dipped into the liquid air, it had not caught fire; $]_{\alpha}[$ but now it exploded, it was consumed so rapidly. $]_{\beta}$

The example is much clearer with now because now helps to separate the time of $\beta$ from the time of $\alpha$ and to enforce a tie between $t_{\beta}$ and $t_{\chi}$.

Now can be omitted in certain subordinating structures: When a clause $\beta$ elaborates on a clause $\alpha$, for example, it is ensured by the semantics of ELAB-

(13) But Rokiroki gripped the strangers wrists so that he could not draw his hatchet. And now he called again to his little daughter... ${ }^{8}$

However, SDRT posits topics for narrations, so now in (13) will have a super-ordinate antecedent determined by the discourse topic. Now, like next and then, is easily used to modify a sentence related to another sentence via NARRATION because all of the sentences that figure in a narration elaborate on the topic event. Because they all elaborate on the topic, they must all share in the topic time, but none can be identical to the topic time because the semantics of NARRATION ensure that there is no temporal overlap between two clauses related by NARRATION. 
ORATION that $t_{\beta}$ is included in $t_{\alpha}$. Sometimes, it is also clear that $t_{\alpha}$ is included in $t_{\beta}$ whether or not $\beta$ is modified by now. This is the case in (3):

(3) [Five months later, $]_{\eta}$ [I sat with her as she lay in bed $]_{\gamma} \ldots$ [I was alone in her bleak room. $]_{\chi}$ [Alone, because there was none of her in it, $]_{\alpha}[$ just a body that (now) held no essence of my mum. $]_{\beta}$

$\beta$ does not figure in a complex subordinate unit, i.e. $\beta$ is not related via a coordinating relation to any other discourse units, and there are no markers such as for example to suggest that $t_{\beta}$ is only properly included in $t_{\alpha}$. Regardless of whether now is used in (3), it is understood that $t_{\beta}$ is $t_{\alpha} \cdot{ }^{9}$ In this case, the requirements of now are already satisfied by $\beta$, so now can be omitted without affecting the truth conditions. This observation can be generalized to other subordinating relations: if a clause $\beta$ is subordinate to a clause $\alpha$ via BACKGROUND or EXPLANATION and $t_{\alpha}$ and $t_{\beta}$ are as close to identical as allowed by the semantics of these relations without now, then the requirements of now are satisfied and now can be omitted without changing the truth conditions for the discourse.

\section{A note about contrast and change of state}

If we remove now from (3), the truth conditions of the example do not change, but something is nonetheless lost. Now suggests that the state described in $\beta$ began recently; the change from the author's mother's body having an essence to its not holding an essence is important for the story the author is recounting and the use of now reinforces this theme. Similarly, in (12), now makes it clear that Darwin did not always have the fear described in the now-modified clause. Now suggests a change in Darwin's thinking and so aids the tie between the now modified clause and its antecedent, which mentions the catalyst for the change in Darwin's thinking. As a final illustration, now in (8) emphasizes that fact that the felt's exploding is a new event and so reinforces the contrast that holds between the now modified clause and the preceding clause.

As Hunter (2010), Lee \& Choi (2009), and Recanati (2004) have observed, now, at least when it modifies past tense clauses, often signals a recent change or a contrast of some sort. In opposition to Hunter (2010) and Recanati (2004), however, I maintain that this effect does not arise from a semantic requirement that the eventuality described by the now-modified clause be contrasted, either explicitly or implicitly, with some other eventuality. Rather, the contrastive feel of so many now examples is a pragmatic effect that arises naturally from the semantics that I have laid out so far together with certain features of a discourse. There are multiple reasons to resist the claim that now requires a contrast or a

9 The chances are high that the author's mother's room contained an essence-less body long before the time at which the author paid the visit under discussion in $\alpha$. Nevertheless, the discourse only demands that $t_{\alpha}$ be the same as $t_{\beta}$. The discourse could be true in a scenario in which the mother's body lost its essence at exactly the moment that the author walked through the door to pay the visit mentioned in $\alpha$. While this scenario is implausible, it is allowed by the discourse structure. 
change of state. First, even in examples that have a contrastive feel, it is often difficult to say in what sense these examples contain a contrast. It is certainly not the case that the now-modified clause must be related to another clause via the CONTRAST relation defined in SDRT, for example. Amongst the English examples that I have discussed in this article, only in (8) would SDRT say that the now-modified clause is an argument for CONTRAST.

Second, there are many examples in which now modifies a past tense clause that do not give rise to a contrastive effect.

(14) [2011 was a great year for computer science.] In attacking the problem of the ambiguity of human language, computer science was now closing in on what researchers refer to as the "Paris Hilton problem". ${ }^{10}$

(15) But Rokiroki, exerting all his strength, gripped the strangers wrists so that he could not draw his hatchet. And now he called again to his little daughter, who stood trembling on the bank above.

In (14), it is obvious from the context that computer science was not closing in on the Paris Hilton problem before whatever time serves as now's antecedent. In this sense, there is a kind of opposition implicit between the time at which computer science is said to have been closing in on the Paris Hilton problem and the times before. Nevertheless, (14) does not have a contrastive feel because the change of state is not at issue in the discourse. Now simply serves to emphasize the period under discussion in the discourse and other times are not relevant. Note that we could replace now in this example with at this time and the implicit opposition between the time of the eventuality described by the now-modified clause and previous times would still be there. Yet we would not for this reason want to build a requirement of contrast into the semantics of at this time. In (15), now again signals a change from one eventuality to another, but again, this is not a reason to argue that now requires a contrast between two times. For one thing, now could not felicitously be replaced by but, a marker for contrast in discourse theories like SDRT. For another, and now could be replaced by next or and then and the discourse would have the same effect of signalling a shift from one eventuality to another. But as with at this time, we would not want to argue that next or then requires a contrast.

The contrastive feel of examples involving past tense uses of now is better explained as a natural consequence of the semantics of now combined with certain features of the discourse in which the now modified clause figures. Now inherits the time of its super-ordinate antecedent. Sometimes this feature of now's semantics allows it to play an indispensable structuring role in a discourse. Other times, now is not needed to structure a discourse, but simply serves to emphasize the temporal relation between the clause it modifies and its antecedent. Now's semantics stop here and will not give rise to a contrastive effect on their own. If, however, within the discourse, the now-modified clause and its antecedent clause fall on one side of a larger contrastive structure, then the fact that now

${ }^{10}$ Variation on example in 'A Fight to Win the Future: Computers vs. Humans,' The New York Times, 14.02.2011. 
emphasizes the temporal relation between the clause that it modifies and its antecedent will naturally give rise to an emphasis on the temporal nature of the contrasted eventualities. In (5) and (8), the now-modified clause enters into a local contrast relation with the previous clause in the discourse. Because the use of now emphasizes the temporal nature of one side of the contrast, the temporal nature of the other side is brought to light. In (3) and (12), the discourse is about a change of a body state and a change in a set of beliefs, respectively. In both cases, the discourse sets up a much higher-level contrastive structure. Again, now serves to emphasize the temporal relations on one side of this structure, which naturally gives rise to a 'then' vs. 'now' reading of the contrastive structure. ${ }^{11}$

\section{References}

Afantenos, S., et al: An empirical resource for discovering cognitive principles of discourse organization: the ANNODIS corpus, Proceedings of LREC 2012 (2012)

Asher, N., Lascarides, A.: The Logics of Conversation. Cambridge University Press (2003)

Banfield, A.: Unspeakable Sentences: Narration and Representation in the Language of Fiction. Routledge \& Kegan Paul, London (1982)

Hunter, J.: Presuppositional Indexicals. Ph.D. thesis, The University of Texas (2010)

Hunter, J., Asher, N.: A presuppositional account of indexicals. In: Dekker, P., Franke, M. (eds.) The Proceedings of the $15^{\text {th }}$ Amsterdam Colloquium. pp. 119-124 (2005)

Kamp, H.: Formal properties of now. Theoria 37, 227-273 (1971)

Kamp, H., Reyle, U.: From Discourse to Logic. Kluwer Academic Publishers (1993)

Kaplan, D.: Demonstratives. In: Almog, J., Perry, J., Wettstein, H. (eds.) Themes from Kaplan. Oxford University Press, USA (1989)

Lee, E., Choi, J.: Two nows in korean. Journal of Semantics 26 (2009)

Maier, E.: Proper names and indexicals trigger rigid presuppositions. Journal of Semantics 23, 253-315 (2009)

Predelli, S.: Utterance, interpretation and the logic of indexicals. Mind $\mathscr{E}$ Language 13(3), 400-414 (1998)

Recanati, F.: Indexicality and context shift (manuscript), Harvard University (2004)

Roberts, C.: Demonstratives as definites. In: van Deemter, K., Kibble, R. (eds.) Information Sharing. CSLI Press (2002)

Rooth, M.: A theory of focus interpretation. Natural Language Semantics 1, 75-116 (1992)

van der Sandt, R.: Presupposition projection as anaphora resolution. Journal of Semantics 9, 333-377 (1992)

Schlenker, P.: Context of thought and context of utterance. Mind $\mathscr{G}$ Language 19(3), 279-304 (2004)

Zeevat, H.: Demonstratives in discourse. Journal of Semantics 16, 279-313 (1999)

11 Another factor that might encourage a contrastive reading of now is focus. Following the work of Rooth (1992), a focused element in a sentence gives rise to a set of alternatives, which in turn gives rise to a contrast. How exactly focus affects the interpretation of now would be an interesting topic for further study, though I doubt that focus affects the fundamentals of the theory that I am presenting here. 